\title{
Models of the Double-mode Cepheids in the Large Magellanic Cloud
}

\author{
J. O. Petersen ${ }^{1}$ and J. Christensen-Dalsgaard ${ }^{2}$ \\ ${ }^{1}$ Theoretical Astrophysics Center, and \\ Niels Bohr Institute for Astronomy, Physics and Geophysics, \\ Astronomical Observatory, Øster Voldgade 3, DK 1350 Copenhagen K, \\ Denmark, (E-mail: oz@astro.ku.dk) \\ ${ }^{2}$ Theoretical Astrophysics Center, and \\ Institute of Physics and Astronomy, Aarhus University, \\ DK8000 Aarhus C, Denmark, (E-mail: jcd@obs.aau.dk)
}

\begin{abstract}
Very recently the MACHO Collaboration published detailed data for 45 double-mode Cepheids in the Large Magellanic Cloud. Here we compare these data with new stellar pulsation models. We conclude that the new period ratios are in good agreement with a metal content $Z=0.005-0.01$ in the Large Magellanic Cloud.
\end{abstract}

\section{Introduction}

The double-mode Cepheids pulsating in two radial modes have been crucial for testing of Cepheid models (e.g., Andreasen \& Petersen 1988, ChristensenDalsgaard 1992). These studies have been based on observations of 15 doublemode Cepheids known in the Galaxy. Very recently the MACHO Collaboration (Alcock et al. 1995) published detailed data for 45 double-mode Cepheids in the Large Magellanic Cloud, 30 oscillating in the fundamental mode and the first overtone and 15 oscillating in the first two overtones.

\section{Model series and comparisons with observations}

We have calculated precise periods of linear, adiabatic oscillations for the fundamental mode and the first few overtones of series of Cepheid models generally situated in the middle of the Cepheid instability strip using OPAL opacities. We use two mass-luminosity relations: ML1 is the BIT mass-luminosity relation (Becker et al. 1977), while ML2 approximately fits calculations including a moderate amount of overshooting from convective cores. Comparing model series with somewhat changed parameters (e.g., increased effective temperature at constant luminosity or a change in the mixing-length parameter, $\alpha$, from our standard value of 2 to 1 ), we find that effects from these changes are minor compared to the effects of changes in the metal content, $Z$, and the mass-luminosity relation. We therefore concentrate on comparing the observed period ratios with results for models with varying $Z$, for the mass-luminosity relations ML1 and ML2. 
For $\Pi_{1} / \Pi_{0}$ we find that the computed period ratios show a substantial dependence on both $Z$ and the $M-L$ relation. The 14 DMCs in the Galaxy correspond to models with $Z$ in the interval $0.01-0.02$, and we cannot distinguish between ML1 and ML2 from the available period ratios. It is interesting that the average $Z$ for the relatively young DMCs in the Milky Way appears to be somewhat smaller than the the solar value of $Z=0.017-0.020$.

The LMC stars are nicely described by models with $Z$ in the range 0.0050.010 , except for one star apparently indicating $Z \simeq 0.015$. For periods larger than about $3 \mathrm{~d}$ (18 stars) we find that $\Pi_{1} / \Pi_{0}$ is mainly determined by $Z$. But for periods smaller than about $3 \mathrm{~d}$ (12 stars) we now find that the change from ML1 to ML2 can have effects on the period ratio that are larger than those of a change in $Z$ by a factor of 2 . Given our lack of knowledge about the $Z$-value, we cannot choose between these possibilities.

LMC contains 15 Cepheids oscillating in the first and second overtones, providing a new test of the theoretical models. For periods smaller than about 1 d (where five stars are observed), we find a separation after $Z$ in the computed $\Pi_{2} / \Pi_{1}$, similar to the separation in $\Pi_{1} / \Pi_{0}$ for $\Pi_{0} \geq 3 \mathrm{~d}$. In the period interval $\Pi_{1}$ $=1.0-1.5 \mathrm{~d}$, where the bulk of the $1 \mathrm{OT} / 2 \mathrm{OT}$ variables are found, the theoretical $\Pi_{2} / \Pi_{1}$ depends sensitively both on $Z$ and the assumed $M-L$ relation. For $\Pi_{1} \geq 1.5 \mathrm{~d}$ we now find that $\Pi_{2} / \Pi_{1}$ is mainly determined by the $M-L$ relation. Thus the accurately known $\Pi_{2} / \Pi_{1}$ is determined by different effects in different period intervals.

\section{Conclusion}

Our results confirm that period ratios for double-mode Cepheid models computed with the OPAL opacities, and for masses corresponding to evolution calculations, are broadly consistent with the observed values.

Comparison of double-mode Cepheids in the Galaxy and the Large Magellanic Cloud shows a difference in the average $\Pi_{1} / \Pi_{0}$ corresponding to a lower heavy-element abundance $Z$, by roughly a factor of 2 , in the LMC, and this result is also in agreement with the observed values of $\Pi_{2} / \Pi_{1}$.

Acknowledgments. We wish to thank Doug Welch for sending us the data for the LMC double-mode Cepheids by e-mail. Support from the Danish National Research Foundation via TAC is gratefully acknowledged.

\section{References}

Alcock, C., Allsman, R.A., Axelrod, T.S., Bennett, D.P., Cook, K.H., Freeman, K.C., Griest, K., Marshall, S.L., Peterson, B.A., Pratt, M.R., Quinn, P.J., Reimann, J., Rodgers, A.W., Stubbs, C.W., Sutherland, W., \& Welch, D.L. 1995, preprint (astro-ph/9411061)

Andreasen, G.K., \& Petersen, J.O. 1988, A\&A, 192, L4

Becker, S.A., Iben, I., \& Tuggle, R.S. 1977, ApJ, 218, 633

Christensen-Dalsgaard, J. 1992, in: "Inside the Stars", IAU Coll. 137, eds. W.W. Weiss, A. Baglin, ASP Conf. Ser., 40, 483 